\title{
Health facilities roles in measuring progress of universal health coverage
}

\author{
Muhiuddin Haider ${ }^{1}$, Emily Vooris ${ }^{2}$, Ananya Krishnan ${ }^{3}$ \\ ${ }^{1,2}$ School of Public Health, University of Maryland, College Park, Maryland, United States \\ ${ }^{3}$ University of Maryland, College Park, Maryland, United States
}

\begin{tabular}{l} 
Article Info \\
\hline Article history: \\
Received Aug 21, 2020 \\
Revised Dec 20, 2020 \\
Accepted Jan 4, 2021 \\
\hline
\end{tabular}

Keywords:

Bangladesh

Global health

Health facilities survey

Universal health coverage

World Health Organization

\begin{abstract}
Outlined in Sustainable Development Goal 3.8, universal health coverage (UHC) ensures all people can access affordable and equitable essential health services without facing economic challenges. Advised by the World Health Organization (WHO), countries can strengthen their health systems and subsequently UHC by establishing a robust health system on a framework of service delivery; health workforce; information; medical products, vaccines and technologies; financing; and leadership and governance. By achieving UHC, countries progress in other health-related goals and provide for healthier children, a stronger workforce and long-term economic development. As announced by Prime Minister Sheikh Hasina in 2011, Bangladesh has remained committed towards UHC through the implementation of programs that increase availability and financial accessibility of essential health services. To produce information regarding their contribution to UHC and specifically the work of its health facilities, Bangladesh produced the 2017 Bangladesh health facilities survey (BHFS). Based on a qualitative analysis, the 2017 BHFS provides substantial information regarding the presence of essential services within different facilities and locations. However, the survey inadequately addresses other components that contribute to availability and accessibility of services, including utilization, patient load, quality of care and financial burden. Subsequently, the 2017 BHFS does not provide a comprehensive evaluation of their health facilities and their contribution to UHC. Arguably, a future survey must address these topics and incorporate a multidisciplinary approach to successfully implement UHC. This approach would incorporate multidisciplinary stakeholders including economists, public health figures and politicians to address challenges such as financial burden, public distrust, and qualified training of providers.
\end{abstract}

This is an open access article under the $\underline{C C B Y-S A}$ license.

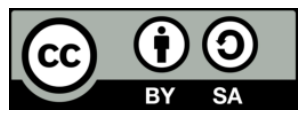

\section{Corresponding Author:}

Muhiuddin Haider

School of Public Health

University of Maryland

College Park, Maryland, United States

Email: mhaider@umd.edu

\section{INTRODUCTION}

Bangladesh remains committed to developing a pathway towards universal health coverage (UHC), an objective to provide appropriate and accessible health services to all individuals without financial burden $[1,2]$. These efforts are reflected in policies and the implementation of programs by the country's pluralistic health system and health facilities. In an effort to analyze the availability and readiness of its health facilities, 
the National Institute of Population Research and Training (NIPORT) produced the Bangladesh Health Facilities Survey 2017 (2017 BHFS) [1]. The survey attempts to provide conclusive nationally representative sample survey on the country's health facilities and the essential services offered within its facilities. The results of the survey have the potential to define future policies, research and strategies for the healthcare system and subsequently influence national health outcomes [3]. This paper uses the 2017 BFHS to analyze Bangladesh's health facilities' contribution to UHC and facilities' potential for improving health outcomes. The paper discusses the information and data provided within the 2017 BHFS and scrutinizes this survey as an insufficient means to examine Bangladesh's progress towards UHC.

\section{The importance of UHC}

Outlined in Sustainable Development Goal 3.8, UHC is characterized by three components that ensure all people can access affordable and equitable health services without facing economic challenges. These components are protection from financial risk or downfall for accessing health services; access to quality health care services; and access to essential medicines and vaccines that are safe, effective, and affordable [2]. Implemented on both an individual and population level, UHC ensures everyone can access quality health services to improve their health while not facing extreme economic consequences. By achieving UHC, countries will make progress in other health-related goals and subsequently provide for healthier children, a stronger workforce and long-term economic development.

\section{WHO framework, SDG indicators, and UHC}

To provide guidance in the implementation of UHC, the World Health Organization (WHO) created the Framework for Action consisting of six building blocks to describe a health systems framework. These six building blocks including service delivery; health workforce; information; medical products, vaccines and technologies; financing; and leadership and governance [2]. Successful health service delivery provides effective, safe, quality personal and non-personal health intervention to the population when and where needed, as efficiently as possible. A well-performing health workforce is responsive, fair and efficiently designed to achieve the best health outcomes possible, given the necessary resources and circumstances. Well-functioning health information systems provide accessible, reliable, and timely information on health determinants, health system performance and health status. A well-functioning health system ensures equitable access to essential medical products, vaccines and technologies of assured quality, safety, efficacy and cost-effectiveness, and their scientifically sound and cost-effective use [2]. Further, successful leadership and governance involves developing strategic policy frameworks and are combined with effective oversight, coalition building, regulation, and attention to system-design [2]. The 2013 World Heath Report also identified essential methods for health systems to support research focused on achieving UHC: setting research priorities, building research capacity, defining norms and standards for research, and translating evidence into practice [4]. These building blocks and practices are crucial to ensure the availability and accessibility of all essential health services [5].

To support the health system framework and monitor progress towards UHC, WHO established two indicators within the sustainable development goals (SDGs). The first indicator is the coverage of all essential health services (SDG indicator 3.8.1). A health system should provide essential health services including reproductive, maternal, newborn and child health (RMNCH), infectious disease, noncommunicable disease, and injuries [6]. These health services should include prevention services in the form of health promotion and illness prevention, treatment services through curative services, rehabilitation and palliation, and coverage in priority global health areas. With good service delivery, a productive and trained health workforce, a well-functioning health information system, adequate medical products, and vaccines and technologies, a country can provide equitable and accessible essential health services.

While establishing and structuring accessible health services is vital to UHC, the financial cost of healthcare on populations is also a crucial component of accessibility. The second indicator of UHC (3.8.2) is defined as the "proportion of the population with large household expenditure on health as a share of total household expenditure or income" [7]. Two thresholds are used to determine whether household expenditures are catastrophic by whether the household expenditure on health is greater than $10 \%$ and greater than $25 \%$ [7]. Large out of pocket payments often pose a difficult challenge for families who must choose between health and other priorities like education or food [7]. However, a successful health financing system and governance can raise adequate funds for health, ensuring populations can utilize needed services without experiencing financial catastrophe associated with healthcare costs. These building blocks protect populations from financial ruin by holding the government accountable for providing the aforementioned accessible and effective healthcare [8].

Through the SDG indicators and the Health System Framework, WHO creates tangible goals for countries to obtain standards of UHC. Further, these frameworks and indicators promote "a common understanding of what a health system is and what constitutes health systems strengthening" [2]. This framework helps clearly define WHO's current priorities and requirements for UHC, which can assist in 
identifying gaps in the current policy. Countries can use these basic guidelines to address their unique healthcare issues and identify gaps in their current system delivery and policies [9]. In fact, many lower- and middle-income countries in Latin America, Africa, and Asia have successfully implemented or are currently implementing policies to achieve UHC through this framework [10-18].

\section{Universal health care in Bangladesh}

For several decades, Bangladesh has remained committed to improving its health care system and essential services. Through support from international donors, the country has implemented a number of plans and policies to successfully adhere to WHO's defined UHC criterion [19]. In 2011, National Health Policy (NHP) was released, comprised of specific policy establishing principles of health designed to produce the most efficient and equal healthcare system possible [20]. The same year, Prime Minister Sheikh Hasina committed to achieving UHC by 2032, outsourcing additional resources to create a feasible plan [21]. These plans are supported and reinforced by three major programs dedicated to improving the health, nutrition, and population sectors through targeted policy development [21]. These policies are further supplemented by simultaneous projects funded by the World Bank and various bilateral organizations that help operationalize and specify the goals of these plans [21].

Bangladesh currently implements these programs and policies under a pluralistic health system [22]. Pluralistic health systems are highly unregulated and consist of multiple private and public key players that provide health care, rather than a regulated organization of providers under one large force such as the government [23]. In Bangladesh, the health delivery chain is an intricate network of providers. The four key players are the government, for-profit private sector, not-for private and non-governmental organization (NGO) sectors, and international development organizations [24]. The Ministry of Health and Family Welfare is the largest employer for health services in Bangladesh. However, there are also many players in the private sector, which accounts for $60 \%$ of health care services [25]. This includes but is not limited to outsourced healthcare providers from international organizations, NGO's, the for-profit private sector, and individual providers [22]. In fact, community-based health workers are often the first point of contact in the health delivery chain for many patients. While health insurance is not easily obtained and accessible, the public health system is highly subsidized and set up to effectively remove the burden of payment for patient care [22]. The structure of this system delivering care is outlined by the aforementioned policy that determines what healthcare truly looks like in Bangladesh.

The pluralistic system is guided by the principles of UHC; to recognize health as a human right, ensure primary and emergency health care for all, increase and expand citizen centric quality health care ensuring equity, enable people to seek healthcare and undertake healthy lifestyles, and improve public health and nutrition [26]. To meet these objectives and provide all essential services, the system is divided into distinct levels of service delivery. The most basic level of primary care consists of upazila health complexes in subdistricts, community clinics in villages, and the Union Health and Family Welfare Centers (UHFWC) at the Union level. The secondary level consists of local maternal and child welfare centers and district hospitals equipped with specialist services such as internal medicine, pathology, obstetrics and gynecology, and surgery to support the primary level providers. The tertiary level encompasses national hospitals, medical universities, specialty hospitals, and medical college hospitals [27]. While these levels do collaborate to care for the population, they each contribute unique services and occupy individual roles in the healthcare system. This delegation of services allows for UHC requirements and responsibilities to be evenly distributed between a multitude of providers based on their accessibility, resources, and expertise.

Bangladesh's commitment to improving its health system has manifested in many successes, most notably the improvement in maternal and child health [27, 28]. From 2010 to 2016, infant mortality had reduced by $6.9 \%$, maternal mortality had reduced by $6.6 \%$, and under 5 mortality had reduced by $1.52 \%$ [26]. A further success emerging as Bangladesh develops and increases prioritization of healthcare is the pioneering of many innovations and healthcare strategies that have been scaled up [29]. Strategies like expanding the broad reach of community healthcare workers to reach all households largely improve access to healthcare and consequently improve health outcomes [29]. Immunization rates, access to clean water, and sanitation have been improving as well [26].

However, Bangladesh's health system is accompanied by a number of unique challenges. Only 2.64 percent of total gross domestic product (GDP) expenditure goes towards health. This is the lowest expenditure percentage in the south Asia region [22]. This economic insufficiency combined with overpopulation and handling such a large population inevitably leads to overcrowding, understaffing, shortage of qualified professionals, and an unequal spread of resources between rural and urban areas [21, 28]. Rural populations account for $62.6 \%$ of the total population, yet still lack funding and care [30]. This is also due to a large problem of absenteeism and low retention rates of physicians in rural areas [31]. Overcrowding and a lack of resources also make Bangladesh a hotspot for communicable diseases, particularly Tuberculosis, and non-communicable diseases like diabetes [6, 32]. A large portion of the 
population also suffers from malnutrition and sanitation issues [29, 33]. Other scholars argue that issues such as public distrust of the health financing system challenge Bangladesh's implementation of UHC [34].

\section{RESEARCH METHOD}

In an effort to evaluate their health facilities and their progress towards UHC, Bangladesh conducted the 2017 Bangladesh Health Facility Survey (2017 BHFS). Several studies have assessed the role of health care facility surveys and evaluated the types of information these surveys gather [35, 36]. Using the data and information collected from health care facility surveys, such as the 2017 BHFS, policy makers and researchers can evaluate the current state of the health system. Subsequently, health facility surveys can define future research opportunities, resource allocation, policy strategies and priority areas for action [37].

The type of data collected through health facility surveys varies and is often reflected in the survey's main objectives [3, 37]. Data can reflect information regarding inputs or structure, processes and/or outcomes. Input or structure data refers to "availability and quantity of inputs" of components such as infrastructure, supplies and equipment, management and information systems [37]. Process or output data refers to activities performed within an intervention. This type of data includes the utilization of services and capacity of care. Lastly, outcome data is a measurement of impact such as health status statistics or estimates of healthy lives per dollar expended [37]. This measurement provides information on the health impact of the overall and individual components of a health system. Collectively, input, process, output, and outcome data can provide performance measures in the form of effectiveness, equity, and efficiency. These performance measures can create a holistic picture of the functionality of a health system.

Using the WHO framework for health systems and the six pillars of health systems, we qualitatively evaluate what information the Bangladesh's Health Facility Survey 2017 (BHFS) provides regarding the availability of essential services and the financing of this system to avoid catastrophic spending. We situate our discussion based on health system inputs such as policies and guidelines, the organization of providers and facilities, and process indicators on the accessibility and quality of services. We connect our analysis to the six pillars of a health system including service delivery; health workforce; information; medical products, vaccines and technologies; financing; and leadership and governance. Subsequently, we evaluate what additional information is needed to adequately evaluate Bangladesh's progress towards UHC.

\section{RESULTS AND DISCUSSION}

Overall, Bangladesh's Health Facility Survey 2017 (BHFS) provides extensive structural and input data to assess the availability of health services across the country. The main objectives of the survey were to assess availability of services; assess preparedness of health facilities; assess service specific readiness; and compare findings among facilities and managing authorities [1]. More specifically, the survey aims to provide information regarding health facilities and their readiness to offer the following health services: child health, family planning, antenatal care, delivery and newborn care, non-communicable diseases, and tuberculosis. Within each health topic, the survey discusses data regarding the availability of services, service readiness, and basic management and administrative systems. Data is compared across facility type, provider type and facility location.

With this breadth of information, we anticipated the BHFS would provide information to analyze the health facility's contribution to UHC in Bangladesh. However, the data did not present such an analysis. Instead, we analyzed what type of information was provided within the BHFS, and what type of information is needed to successfully analyze the position of UHC in Bangladesh. Categorically, we interpreted these inputs, outputs and outcomes based on Kurk and Freedman's [38] health facility performance measures as shown in Figure 1.

Kurk and Freedman's [38] framework outlines how the flow of inputs and outputs that contribute to a facility's effectiveness, equity, and efficiency of services. Additionally, the framework emphasizes multisectoral involvement in research and essential services within UHC. Using this framework, our analysis resulted in the following discussion of BHFS' contribution of information on organization, guidelines and policy, availability, and finance of Bangladesh's health facilities 


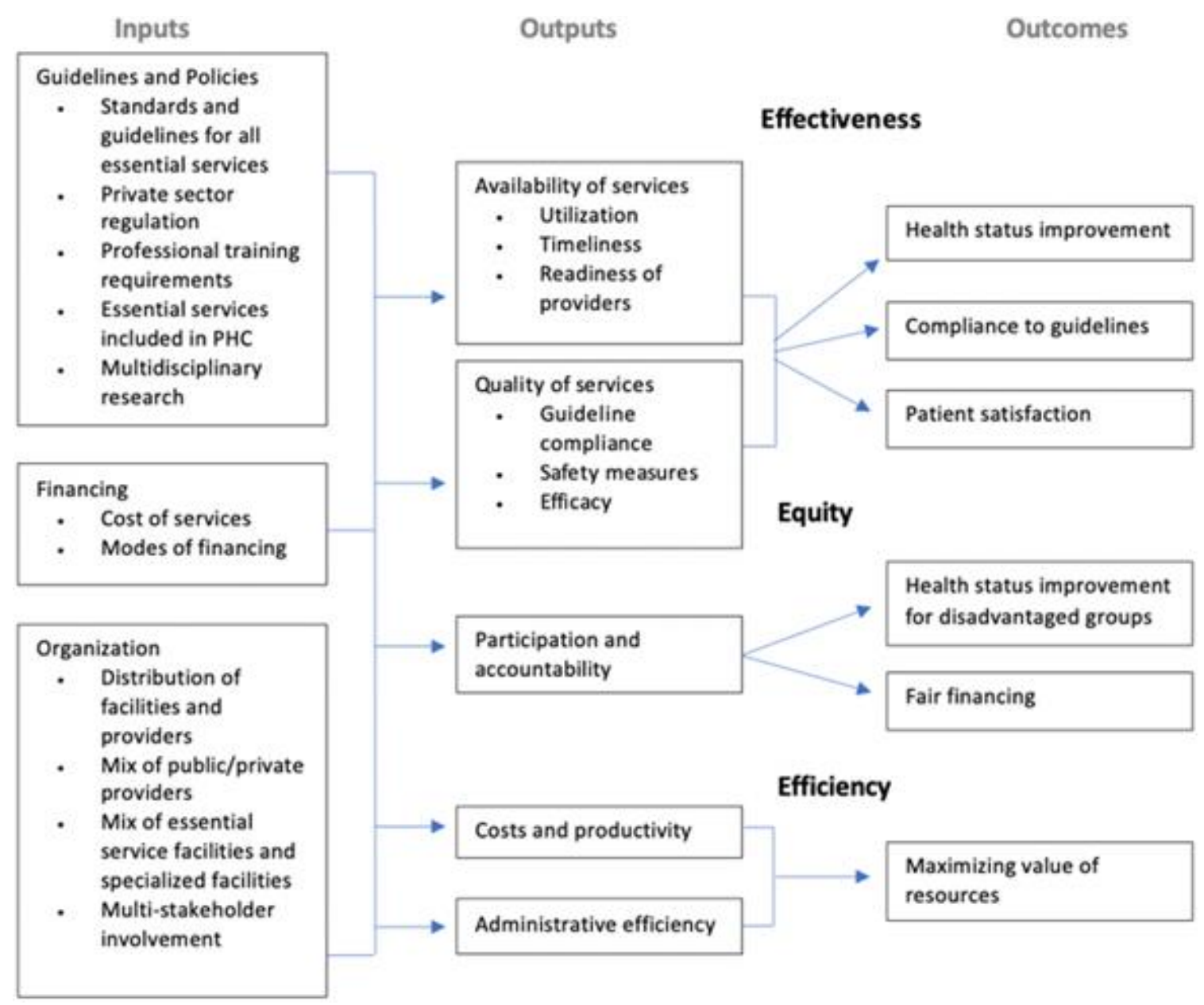

Figure 1. Health facility performance measures, sourced from Kurk and Freedman [38]

\subsection{Organization}

In each section, the 2017 BHFS evaluates indicators of availability and readiness of its health services compared by facility and provider type. The survey provides an in-depth overview of the health system in Bangladesh and the organization of the management structure of health facilities under directorate general of health services (DGHS). By distinguishing between facility type and location, the survey provides data on the locality of particular services and highlights what types of facilities take responsibility for particular services. For example, mother and child welfare centres (MCWC) are the most prepared facilities for family planning while district hospitals and private hospitals have the lowest levels of readiness and availability of family planning services. In contrast, private hospitals are the most likely to have essential equipment and services for treating non-communicable diseases such as cardiovascular disease, and chronic respiratory disease. Researchers can effectively use this type of organizational data to make conclusions on the private/public provider ratios, the distribution of facilities in relation to mapping and the census. Subsequently such data can be used to suggest whether the facilities are located in accessible locations to disadvantaged groups.

The BHFS could have provided some additional information regarding the organization of its facilities. For example, data is often presented in a form of percentages for comparison. This can be misleading when only a few facilities within that particular facility category were interviewed. Additionally, the survey could have elaborated on the division of service delivery levels and the distribution of facilities by district.

\subsection{Guidelines and policy}

Structural inputs into a health care system, such as policies, guidelines, and strategies, create a framework for the collective functioning of a health system. These policy frameworks contribute to leadership and governance, a pillar of WHO's health system framework, and ensure that standard quality care 
is provided. The 2017 BFHS provides discussion of national policies and specific guidelines for a few of its services areas. However, more information could be provided to strengthen its results.

The BFHS successfully articulates national guidelines and policy for a few of its essential health services, mainly child health, delivery/newborn care and tuberculosis. Specifically, the survey discusses the adoption of an integrated management of childhood illness strategy (IMCI) as advised by the WHO. Within this strategy, health care providers should use appointments to evaluate current health, underlying problems as well as preventive interventions to prevent future illness [1]. As a high burden country for tuberculosis, Bangladesh also established the National TB Control Program (NTP) [39]. This program utilizes a model of directly observed treatment (DOTS) that outlines five necessary guidelines including secure political commitment and sustained financing, early case detection and diagnosis, standardized treatment, effective drug supply and management, and monitoring and evaluating performance [40]. The survey also outlines policies in place to improve Delivery and Newborn Care. Bangladesh has developed a "Promise Renewed Declaration: Bangladesh Call to Action 2013" policy and included a National Newborn Health Program (NNHP) in the 4th Health, Population and Nutrition Sector Development Program (HPNSDP). A Maternal, Neonatal, Child, and Adolescent Health (MNC\&AH) plan is known as 'Bangladesh Every Newborn Action Plan' (BENAP) has also been created and operationalized under the HPNSDP [1]. The BHFS describes the specific goals these plans aim to achieve in regards to Delivery and Newborn Care improvement. By discussing the results of the survey in relation to established policy guidelines, the survey creates a standard for health facilities to achieve. The survey also anchors their findings based on existing policy and governance, highlighting the interplay of the health system pillars.

In several sections, the BHFS minimally discusses guidelines and policies for particular services. Specifically, the survey lacks information regarding policies and guidelines for the diagnosis and treatment for various non-communicable diseases including diabetes, cardiovascular disease, chronic respiratory disease and cervical cancer. It only briefly mentioned policies such as the National Population Policy (MOHFW 2012) and the 4th Health, Population and Nutrition Sector Development Program (HPNSP) created to improve family planning services. The survey does not provide information regarding guidelines or policies for antenatal services. The lack of discussion of these guidelines suggests such policies are not established.

Health facilities also need to ensure their providers adhere to guidelines, attend consistent training to ensure compliance and have adequate equipment and medications to provide quality care [37]. The survey discusses these elements through their evaluation of readiness to provide particular health services. Specifically, the survey defines readiness by the facilities' stock of equipment, medicines and whether providers are in compliance with health service guidelines. In many health service categories, the survey used WHO guidelines and indicators as a reference point. However, while readiness was determined based on WHO tracer indicators, the survey often modified indicators to be "less restrictive and context appropriate" [1]. By modifying these indicators, the survey makes it more difficult to effectively compare the data to WHO standards.

Overall, training and compliance to guidelines remained low within each health service type. Urban areas were more likely to have adequate training within their facilities than rural facilities. To evaluate facilities' readiness to provide child health services, the survey evaluated the presence of IMCI guidelines, IMCI trained staff, adequate equipment and medicine. While the country has robust policies matching WHO recommendations, only 5\% of all health facilities have all 10 items within the IMCI categories [1]. Antenatal care readiness was determined by availability of items and equipment. Overall "only $4 \%$ of facilities are at the level of readiness necessary to provide quality ANC services" [1]. Similarly, readiness for delivery and newborn services was determined based on facility guidelines, trained staff, and specialized equipment. 6 out of 10 health facilities were able to offer quality delivery services [1]. Health services with established guidelines and policies, such as tuberculosis services, had much higher rates of guideline compliance.

Overall, the survey openly discusses the importance of training and guideline compliance. The data shows this type of readiness could be improved within the health facilities. However, the survey could have provided additional information regarding who was receiving training and whether this information was subsequently disseminated within the facilities.

\subsection{Availability of services}

The 2017 BHFS aims to evaluate the availability of health services and its individual health facilities. For this survey, availability is defined as the presence of the health service at the health facility [1]. Most data provided on availability was based on whether the particular service was offered at a facility on the day the survey was administered. Using this definition of availability, readers can evaluate differences between facilities and what services they offer. For example, based on the BHFS, child health services are available across all facilities. However, public facilities and NGO facilities provide child growth monitoring 
at higher rates than in private facilities. Additionally, although 9 in 10 of all facilities provide child vaccination, only $7 \%$ of these facilities are private [1]. Further, urban facilities are less likely to have child services. This suggests the availability of child health services is based on location and type of facility.

In a few sections, the BHFS further defines their definition of availability. In their evaluation of family planning services, the survey makes a distinction between providing family planning methods and offering family planning services [1]. The latter includes facilities that provide, prescribe or counsel family planning methods but may refer clients to other facilities for accessibility to those methods or medications. Thus, the statistic that about 9 in 10 of all health facilities offer modern methods of family planning services does not guarantee clients receive the method they need [1]. This distinction is significant as the need to visit another facility could further impede a client's ability to utilize family planning methods in a timely and economically feasible manner.

However, these definitions of availability defined by the presence of a service does not account or address the timeliness or utilization of the health service which helps further define whether the health service is accessible. Delayed diagnosis and treatment of particular health issues can cause additional adverse health outcomes. Based on the BHFS, tuberculosis diagnosis, treatment and/or treatment follow ups are provided at $90 \%$ of district hospitals and $98 \%$ of upazila health complexes (UHC) [1]. However, another study revealed an average health system delay in the management of tuberculosis patients to be 68.5 days [37]. While facilities may be providing services, more information regarding timeliness of services would provide more tangible evidence of the quality and efficiency of the services within these facilities.

Within the section on antenatal health services, the survey describes the percentage of women using services during pregnancy based on data from the 2017 Bangladesh Demographic and Health Survey (BDHS). The results revealed that $82 \%$ of women age 15-49 with a live birth in the 3 years preceding the survey received ANC from a medically trained provider [1]. Additionally, "the 2017 BDHS results showed that only $43 \%$ of rural women, as compared with $59 \%$ of urban women, had at least four ANC visits" [1: 94]. With these statistics, the BFHS is indicating the utilization of antenatal care services. This additional information is necessary to categorize the availability of services further. Additionally, the data was compared to previous survey results from 2014 and 2011, indicating an increase in utilization of antenatal health services.

There is no discussion or data provided regarding the utilization or timeliness of child health services, non-communicable disease, or tuberculosis. The frequency of family planning services was mentioned; "nearly $80 \%$ of facilities reported that they provide FP services every day" [1]. However, this information was not expanded on.

While the 2017 BFHS addresses the availability of services based on the presence of individual health services, the survey did not address other factors that contribute to the accessibility of these health services. Utilization and timeliness of the health services should be discussed. Further, there is no data collected regarding patient load at the facilities or quality of care from the perspective of the patient. As mentioned by Joarder et al. [22] and Andaleeb et al. [41] low utilization of health facilities and increased patient to provider ratios have historically impacted Bangladesh's ability to provide essential services. Furthermore, data regarding quality of care can inform the efficiency, equity, and effectiveness of the health care system.

\subsection{Financing}

As an integral part of SDG 3.8.2 and WHO's Health System Framework, a well-functioning health financing system provides funds for health services that ensure all individuals can access and use services without impoverishment. The 2017 BHFS does not provide any information regarding financing or funding for any health service. The only information discussed is in reference to tuberculosis. Specifically, as part of the NTP, "diagnostic and treatment services are available free of charge in public and private facilities throughout the country" [1]. Without this essential information regarding other health services, it is unclear whether improvements have been made towards UHC specifically in indicator 3.8.2. Additionally, information regarding financial accessibility would provide information regarding the efficiency and equity of the health services provided.

\subsection{Inconsistencies}

The survey contains various inconsistencies and gaps in data required for optimal comprehension. Firstly, there are inconsistencies in labeling data throughout the chapter. Some data are cited in percentages while other data within the same chapter are cited as fractions. While this is a minor issue, it lowers the credibility and standard of the work as a whole. Additionally, the translation of data from tables to text within each chapter is inadequate. While the tables often provide sufficient explanation of survey data collected, this is often not expressed in the chapter where graphs are explained by vague statements. For example, first aid 
signal functions for obstetric and newborn care; grouped as BEmONC, EmONC, and CEmONC were discussed in the Delivery and Newborn Care section [1]. However, the signal functions were later listed in the data table without reference to BEmONC, EmONC, or CEmONC, creating confusion regarding the significance of these signal functions and their connection to newborn health care. There are also inconsistencies between chapters regarding the balance between text and graphs, as well as which data is graphically represented. The Delivery and Newborn Care chapter is text heavy and thoroughly explains the data collected with fewer graphs, while chapters like Family Planning have many visuals but more vague textual descriptions. The graphs are condensed from data tables and are often missing valuable clarification and supplemental information from the original tables. Additionally, certain chapters like Delivery and Newborn Care exclude Community Clinics from certain data collected in the chapter without fully rationalizing this decision.

\subsection{Improvements to survey methodology}

The BHFS could benefit from improvements to the methodology of data collection. The survey does not distinguish between types of staff surveyed. This creates possible bias as different staff in facilities have different roles, training, and knowledge of the facility. If all the physicians with training are occupied when the data was collected, then the data will only reflect the training of less qualified healthcare workers, skewing the data. There is also no clarification on whether data collected is based on time of survey or at any point in time. This is especially pertinent for availability of equipment and services. It is unclear whether equipment was not available at the time of the survey or never available. However, this clarification does exist for training. Data is collected for training within the past 24 months as well as training at any time. Additionally, there are arbitrary cutoffs for certain data. For example, four items for infection control is considered adequate, despite these items being basic necessities like running water and gloves. Furthermore, the survey does not emphasize the impact of quantity of facilities when compared to the availability of services provided. There are many community clinics and few districts and upazila public facilities, yet the data reveals that these few public facilities are often better prepared in regard to availability and readiness. There are only four district hospitals surveyed, yet these facilities are often used as examples of high percentages of availability. This manipulation is misleading and creates the false perception that facilities with high percentages of availability and training are common.

\subsection{Successes}

Although the BFHS has elements that can be improved, it does contain positive aspects that should continue to appear in future health facilities surveys. The survey acknowledges shortcomings and required areas of improvement within each aspect of UHC. The visuals provide comparisons between the 2017 and 2014 surveys to show changes in healthcare in Bangladesh over a three-year time period. Additionally, despite the data revealing the lack of training among providers, many chapters clearly explain the significance of health training and break down the different requisites of training based on health service. Training, experience, and knowledge of standards is especially crucial in neonatal care, as providers cite these factors as deterrents to providing care [42]. Certain sections also provide recommendations on how to achieve UHC and improve categories with low availability or readiness. Most importantly, the data tables provide a comprehensive explanation of what data is being measured, along with clarifications and further explanations for graphs throughout the chapter. The tables are organized based on urban and rural areas, specific geographic divisions, and types of facilities. The clear organization and thorough breakdown of these tables is the heart of the survey data and should be given further significance in future survey reports

\section{CONCLUSION}

The 2017 BHFS highlights the availability of services based on the presence of health services within different facilities in Bangladesh. Overall, the survey provides substantial information on the presence of health services which allows research to evaluate differences between facilities and the types of services they offer. However, this information is mostly infrastructural data and does not sufficiently address information regarding patient load, timeliness, utilization of services or service financing. Subsequently, it is difficult to assess availability and effectiveness of services, and whether individuals can access services without contributing to catastrophic spending on health. Without this information, policy makers and researchers will find it difficult to evaluate the system's progress towards UHC and WHO's indicators of health services.

In the future, Bangladesh should conduct an additional survey to evaluate the progress of its health facilities. This future survey must include data regarding timeliness of services, quality of care, patient load and the health financial system. This will require more detailed definitions of availability and services 
provided and clarifications in any discrepancies in collected data. The discussion of the data can be strengthened by further mention of policies followed within each health service and additional procedures followed to increase access to disadvantaged groups. Additionally, Bangladesh could benefit from a multidisciplinary research approach to UHC. Primarily scientific data-based research could help inform and support policy quantitatively with evidence on the impact of current healthcare policies and practices on the population. Medical professionals and scientists could provide crucial information on medical conditions, innovative solutions to public health issues, and necessary considerations when providing care. An alternative approach could involve policymakers and economic experts researching the financial burden of various healthcare policies to create the most advantageous policies. An anthropological social approach to UHC could take the form of ethnographic studies and would humanize the impacts of health policy, helping to advocate for the diverse population when creating policy. The survey would also benefit from recommendations to achieve UHC within each section. These multidisciplinary perspectives could provide recommendations on achieving UHC within each essential service. With this additional information and evaluation provided in a future BFHS, researchers and policy makers can make a more adequate assessment of the country's progress towards UHC and SDG indicators. Further, Bangladesh can provide more comprehensive and effective solutions to achieving UHC efficiently.

\section{REFERENCES}

[1] National Institute of Population Research and Training (NIPORT) and ICF. Bangladesh Health Facility Survey 2017. Dhaka, Bangladesh: NIPORT, ACPR, and ICF, 2019.

[2] World Health Organization. 2007. Everybody's Business: Strengthening Health Systems to Improve Health Outcomes: WHO's Framework for Action. World Health Organization: Geneva.

[3] A. Edward, et al., A comparative analysis of select health facility survey methods applied in low and middle income countries [working paper WP-09-11]. Chapel Hill, NC: MEASURE Evaluation, 2009.

[4] C. Dye, J. Reeder, and R. Terry, "Research for Universal Health Coverage," Science Translational Medicine, vol. 5, no. 199, pp. 199ed13-199ed13, 2013.

[5] T. Manyazewal, "Using the World Health Organization health system building blocks through survey of healthcare professionals to determine the performance of public healthcare facilities," Archives of Public Health, vol. 75, no. $1,2017$.

[6] World Health Organization and the World Bank. 2015. Tracking Universal Health Coverage: first global monitoring report. World Health Organization: Geneva.

[7] World Health Organization. 2018. Monitoring Sustainable Development Goals-Indicator 3.8.2. World Health Organization: Geneva.

[8] J. A. M. Khan, S. Ahmed, and T. G. Evans, "Catastrophic healthcare expenditure and poverty related to out-ofpocket payments for healthcare in Bangladesh - an estimation of financial risk protection of universal health coverage," Health Policy and Planning, vol. 32, no. 8, pp. 1102-1110, 2017.

[9] J. E. Ataguba and M.-G. Ingabire, "Universal Health Coverage: Assessing Service Coverage and Financial Protection for All," American Journal of Public Health, vol. 106, no. 10, pp. 1780-1781, 2016, doi: https://doi.org/10.2105/AJPH.2016.303375

[10] E. Barasa, P. Nguhiu, and D. Mcintyre, "Measuring progress towards Sustainable Development Goal 3.8 on universal health coverage in Kenya," BMJ Global Health, vol. 3, no. 3, pp. 1-14, 2018.

[11] L. de Andrade, et al., "Social determinants of health, universal health coverage, and sustainable development: case studies from Latin American countries," The Lancet, vol. 385, no. 9975, pp. 1343-1351, 2015.

[12] A. Maeda, et al., "Universal health coverage for inclusive and sustainable development a synthesis of 11 country case studies," Washington, DC: World Bank, 2014

[13] Q. Meng and L. Xu, "Monitoring and Evaluating Progress towards Universal Health Coverage in China," PLOS Medicine, vol. 11, no. 9, p. e1001694, 2014.

[14] V., Tangcharoensathien, et al., "The Political Economy of UHC Reform in Thailand: Lessons for Low-and MiddleIncome Countries," Health Systems \& Reform, vol. 5, no. 3, pp. 195-208, 2019.

[15] A. Alebachew, L. Hatt, and M. Kukla, "Monitoring and Evaluating Progress towards Universal Health Coverage in Ethiopia," PLoS Medicine, vol. 11, no. 9, p. e1001696, 2014.

[16] A. Somanathan, H. L. Dao, and T. V. Tien, "Integrating the Poor into Universal Health Coverage in Vietnam," UNICO Studies Series; No. 24. World Bank, Washington DC, 2013.

[17] X. Aguilera, et al., "Monitoring and Evaluating Progress towards Universal Health Coverage in Chile. PLoS Medicine," vol. 11, no. 9, p.e1001676, 2014.

[18] M., Barreto, et al., "Monitoring and Evaluating Progress towards Universal Health Coverage in Brazil," PLoS Medicine, vol. 11, no. 9, p.e1001692, 2014.

[19] A. Adams, et al., "Innovation for universal health coverage in Bangladesh: a call to action," The Lancet, vol. 382, no. 9910, pp. 2104-2111, 2013.

[20] M. Haque and M. E, Murshid, "Hits and Misses of Bangladesh National Health Policy 2011," Journal of Pharmacy And Bioallied Sciences,” vol. 12, no. 2, p. 83, 2020, doi: 10.4103/jpbs.jpbs_236_19.

[21] S. El-Saharty, et al., "The path to universal health coverage in Bangladesh: bridging the gap of human resources for health," Washington, DC: World Bank Group, 2015, doi: 10.1596/978-1-4648-0536-3. 
[22] T. Joarder, T. Z. Chaudhury, and I. Mannan, "Universal Health Coverage in Bangladesh: Activities, Challenges and Suggestions," Advances in Public Health, 2019, https://doi.org/10.1155/2019/4954095

[23] S. Ahmed, et al., "Harnessing pluralism for better health in Bangladesh," The Lancet, vol. 382, no. 9906, pp. 1746$1755,2013$.

[24] S. Ahmed, et al., "Bangladesh Health System Review," Health Systems in Transition, vol. 5, no. 3, 2015.

[25] K. Alam and S. Ahmed, "Cost recovery of NGO primary health care facilities: a case study in Bangladesh," Cost Effectiveness and Resource Allocation, vol. 8, no. 1, pp. 1-10, 2010

[26] Center for Research and Information, Bangladesh Towards Better Healthcare. CRI, Center for Research and Information, 2018.

[27] "Healthcare Services for All: the Bangladesh Story | CRI," 02-Oct-2014. [Online]. Available: https://cri.org.bd/2014/10/02/healthcare-services-for-all-the-bangladesh-story.

[28] R. Govindaraj, et al., Health and nutrition in urban Bangladesh: social determinants and health sector governance. Washington, D.C.: World Bank Group, 2018, doi:10.1596/978-1-4648-1199-9.

[29] A. M. R. Chowdhury, et al., "The Bangladesh Paradox: Exceptional Health Achievement despite Economic Poverty," The Lancet, vol. 382, no. 9906, pp. 1734-45, 2013, https://doi.org/10.1016/s0140-6736(13)62148-0.

[30] The World Bank, 2020. Rural Population (\% Of Total Population) - Bangladesh | Data. [online] Data.worldbank.org. Available at: 〈https://data.worldbank.org/indicator/SP.RUR.TOTL.ZS?locations=BD>

[31] E. Darkwa, et al., "A qualitative study of factors influencing retention of doctors and nurses at rural healthcare facilities in Bangladesh," BMC Health Services Research, vol. 15, pp. 1, pp. 1-12, 2015.

[32] M. Abdur Rahim, "Diabetes in Bangladesh: Prevalence and Determinants," Thesis, Institute of General Practice and Community Medicine, 2002.

[33] United Nations Children's Fund UNICEF., 2007. The state of the world's children 2008: Child Survival. UNICEF: New York.

[34] N. Ahmed, et al., "Reaching the Unreachable: Barriers of the Poorest to Accessing NGO Healthcare Services in Bangladesh," Journal of Health Population and Nutrition, vol. 24, no. 4, pp. 456-466, 2006.

[35] B. Meessen, et al., "Composition of pluralistic health systems: how much can we learn from household surveys? An exploration in Cambodia," Health Policy and Planning, vol. 26, Suppl. 1, pp. i30-i44, 2011.

[36] World Health Organization. 2003. Health Facility Survey: Tool to evaluate the quality of care delivered to sick children attending outpatient facilities. World Health Organization: Geneva.

[37] L. Magnus and A. Wagstaff, Health facility surveys: an introduction. Washington, D.C.: World Bank, Development Research Group, Public Services, 2003.

[38] M. E. Kruk, and L. P. Freedman, "Assessing health system performance in developing countries: A review of the literature," Health Policy, vol. 85, no. 3, p. 265, 2008.

[39] K. Ehsanul Huq, et al., "Health seeking behaviour and delayed management of tuberculosis patients in rural Bangladesh," BMC infectious diseases, vol. 18, no. 1, p. 515, 2018, doi: https://doi.org/10.1186/s12879-018-3430-0.

[40] World Health Organization Bangladesh, USAID and TB CARE II Bangladesh, 2011. National Guidelines for Tuberculosis Infection Control.

[41] S. S. Andaleeb, N. Siddiqui, and S. Khandakar, "Patient satisfaction with health services in Bangladesh," Health Policy and Planning, vol. 22, no. 4, pp. 263-273, 2007, doi: https://doi.org/10.1093/heapol/czm017.

[42] S. Zaman, et al., "Management of Newborn Infection: Knowledge and attitude among health care providers of selected sub-district hospitals in Bangladesh," International Journal of Perceptions in Public Health, vol. 1, no. 2, pp. 127-132, 2017. 